\title{
Interactive comment on "High-resolution global atmospheric moisture connections from evaporation to precipitation" by Obbe A. Tuinenburg et al.
}

\section{Ruud van der Ent \\ r.j.vanderent@tudelft.nl}

Received and published: 2 September 2020

This paper presents a valuable contribution to the field of moisture recycling. Making the source code and the data openly available is very useful for other researchers interested in the application of these data. Being a co-author myself of a recently published similar dataset, also in ESSD (Link et al., 2020), I warmly welcome alternative datasets, which also give a sense to people outside the field what the general uncertainty is associated with different tracking methods and different data. The fact that the authors were able to provide the data on a relatively high $0.5^{\circ}$ resolution highlights the efficiency of their code for calculations in case there is a single grid cell as source 
That being said, I have one point of concern which has not been brought up by the reviewers so far. Figure 8 shows precipitation as calculated from Utrack (Fig. 8a) and the differences with ERA5 (Fig. 8b). First, I highly suspect either some typos or a calculation error in the postprocesing in Fig. 8a because global average precipitation should be in the range of $2-3 \mathrm{~mm} /$ day, which is thus 1 full order of magnitude higher than the scale in Fig. 8a. Second, I found the results in Fig. 8b difficult to interpret, because it is unclear what formula was exactly used to calculate the error and the logaritmic scale is not intuitive. I suspect the formula to be $\log _{10}\left(P_{\mathrm{ERA}}-P_{\mathrm{Utrack}}\right.$, which means that, for example, a difference of $0.1 \mathrm{~mm} /$ day becomes -1 and a difference of $1 \mathrm{~mm} /$ day is 0 . A difference of 0.1 or $1 \mathrm{~mm} /$ day can be already a significant percentage of the total precipitation in some areas and this way of displaying the error does not allow to see whether there is a consistent under/overestimation of Utrack with respect to ERA5 or not. Moreover, it is unclear whether the differences are calculated on a daily/monthly/yearly/total time range scale, which would also influence the difference. In my opinion it would make sense if the authors would display two panels of relative and absolute error (with a diverging color scheme) and make it clear on which time scale these differences have been calculated. Besides, it would be useful if they would provide a global/land/ocean average error in order to identify whether there is a systematic bias or not, which might possibly explain why the recycling values found by the authors are relatively high compared to most previous research. Perhaps displaying the data in this way would simply confirm the authors' claim that the differences are small, but at least it will be easier to confirm for an independent reader.

Let me stress that it is very much appreciated that the authors bothered to calculate and show the errors in precipitation between ERA5 and Utrack in the first place, which is something I have not seen openly communicated in any other work with Lagrangian tracking schemes. I am convinced that the authors would be able to address my concern and I strongly support publication of this paper.

Interactive comment
Printer-friendly version

Discussion paper 
Interactive comment on Earth Syst. Sci. Data Discuss., https://doi.org/10.5194/essd-2020-89, 2020.

\section{ESSDD}

Interactive comment 